PROCEEDINGS OF THE

AMERICAN MATHEMATICAL SOCIETY

Volume 129, Number 12, Pages 3757-3758

S 0002-9939(01)06330-4

Article electronically published on June 27, 2001

\title{
A PROBLEM OF PRESCRIBING GAUSSIAN CURVATURE ON $S^{2}$
}

\author{
SULBHA GOYAL AND VINOD GOYAL
}

(Communicated by David S. Tartakoff)

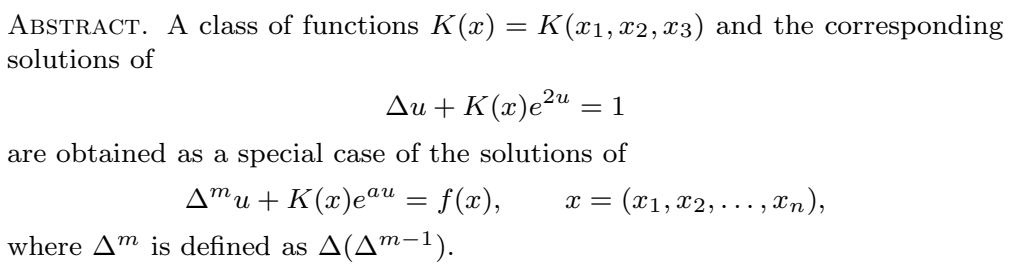

On the two-sphere $S^{2}=\left\{x \in R^{3} \mid x_{1}^{2}+x_{2}^{2}+x_{3}^{2}=1\right\}$ with metric $d s_{0}^{2}=d x_{1}^{2}+$ $d x_{2}^{2}+d x_{3}^{2}$, if the metric is conformally changed to $d s^{2}=e^{2 u} d s_{0}^{2}$, then the Gaussian curvature $K(x)$ of the new metric is determined by the equation

$$
\Delta u+K(x) e^{2 u}=1, \quad x \in S^{2},
$$

where $\Delta$ denotes the Laplacian relative to the metric $d s_{0}^{2}$.

The question raised by L. Nirenberg is: Which functions $K(x)$ can be prescribed so that (1) has a solution $u$ ?

Integrating (11) over the whole sphere, we get

$$
\int_{S^{2}} K e^{2 u} d \mu=4 \pi
$$

where $d \mu$ denotes the surface measure on $S^{2}$. Thus, an obvious necessary condition is that $K$ must be positive somewhere. Another necessary condition was found by Kazdan and Warner [3] via integration by parts. Moser [4] proved that if $K$ is an even function on $S^{2}$, then (1) has a solution. In [1, Cheng and Smoller considered the case of rotationally symmetric functions $K$.

The purpose of this note is to show that a class of functions $K$ and the corresponding solutions can be produced by means of elementary arguments. We first prove the following result:

Theorem 1. If $a>0$ is an arbitrary constant and the functions $K(x)$ and $f(x)$ are positive and such that

$$
\Delta^{m} \ln \left(\frac{K(x)}{f(x)}\right)=0, \quad x=\left(x_{1}, x_{2}, \ldots, x_{n}\right),
$$

Received by the editors December 20, 2000.

2000 Mathematics Subject Classification. Primary 35J30, 35J60; Secondary 31B30.

Key words and phrases. Laplace operator, Gaussian curvature, conformally equivalent, metric. 
then the solution of

$$
\Delta^{m} u+K(x) e^{a u}=f(x)
$$

is given by

$$
u=\frac{1}{a} \ln \left(\frac{f(x)}{K(x)}\right) .
$$

Proof. We divide (3) by $f(x)$ and write it as

$$
\frac{1}{f(x)} \Delta^{m} u+e^{a u+\ln \left(\frac{K(x)}{f(x)}\right)}=1 .
$$

Now by the change of variables

$$
a u+\ln \left(\frac{K(x)}{f(x)}\right)=\nu
$$

and with the help of (2), we obtain

$$
\Delta^{m} u=\frac{1}{a} \Delta^{m} \nu
$$

Thus the equation (5) reduces to

$$
\frac{1}{a f(x)} \Delta^{m} \nu+e^{\nu}=1 .
$$

Clearly, $\nu=0$ is a solution of (8) . We then get from (6)

$$
u=\frac{1}{a} \ln \left(\frac{f(x)}{K(x)}\right) \text {. }
$$

This completes the proof.

If in Theorem 1 we choose $m=1, a=2, n=3$ and $f(x)=1$, then we have

Theorem 2. If the function $K(x)=K\left(x_{1}, x_{2}, x_{3}\right)$ is positive and $\ln (K(x))$ is harmonic, then $u=\frac{1}{2} \ln \left(\frac{1}{K(x)}\right)$ is a solution of (11).

Remark. Evidently $u=\frac{1}{a} \ln \left(\frac{f(x)}{K(x)}\right)$ is also a solution of the nonlinear Dirichlet problem $\Delta u+K(x) e^{a u}=f(x)$ in $D$ and $u=0$ on $\partial D$ as long as $f(x)=K(x)$ on $\partial D$ and $\ln \left(\frac{K(x)}{f(x)}\right)$ is harmonic in $D$.

\section{REFERENCES}

1. K. Cheng and J. Smoller, Conformal metric with prescribed Gaussian curvature on $S^{2}$, UAB International Conference on Differential Equations and Mathematical Physics (Abstracts), March 15-21, 1990, p. 48; Trans. Amer. Math. Soc. 336 (1993), 219-251. MR 93e:53044

2. V. B. Goyal, Remark on a paper of Cheng and Smoller, Proc. Amer. Math. Soc. 113 (1991), 795-797. MR 92b:58243

3. J. Kazdan and F. Warner, Curvature functions for compact 2-manifolds, Ann. of Math. (2) 99 (1974), 14-47. MR 49:7949

4. J. Moser, On a non-linear problem in differential geometry, Dynamical Systems, M. Peixoto (ed.), Academic Press, New York, 1973. MR 49:4018

Department of Mathematics, Tuskegee University, Tuskegee, Alabama 36088

Department of Mathematics, Tuskegee University, Tuskegee, Alabama 36088 